\title{
A Decision-Making Framework for Safe Operations of Unmanned Aerial Vehicles in Urban Environments
}

\author{
Marcos Quiñones-Grueiro ${ }^{1}$, Timothy Darrah ${ }^{2}$, Gautam Biswas ${ }^{3}$, and Chetan Kulkarni ${ }^{4}$ \\ 1,2, 3 Vanderbilt University, Nashville TN, 37209 USA \\ marcos.quinones@vanderbilt.edu \\ timothy.s.darrah@vanderbilt.edu \\ gautam.biswas@vanderbilt.edu \\ ${ }^{4}$ KBR. Inc., NASA Ames Research Center, Moffett Field, CA, 94035 \\ chetan.s.kulkarni@nasa.gov
}

\begin{abstract}
This paper develops an offline decision-making framework to support safe urban operations of individual unmanned aerial vehicles (UAV) flights. The core of the proposed framework is the analysis of the probability of mission failure and the corresponding risk of flight as a function of two factors: (1) collision with obstacles, and (2) crashes attributed to the degraded state of the vehicle. The risk computation is associated with specific trajectories defined by a set of way points. Our experimental studies consider a UAV mission fails when (1) it collides with other objects, or (2) the battery charge is depleted below a threshold. The decision making system automatically selects the mission plan that minimizes risk of flight by considering the state of the vehicle, the environmental conditions, and a map of the environment.
\end{abstract}

\section{INTRODUCTION}

The use of Unmanned Aerial Vehicles (UAVs) is increasing at unprecedented rates across across a wide range of applications that include surveillance, package delivery, photography, cartography, remote sensing, agriculture, military missions, and more ${ }^{1}$. The FAA passed regulations in 2016 that authorized the commercial use of UAVs. As adoption and use of drones increase, so does the risk of collisions and mishaps that can result in a loss of money, time, productivity, or most important, human lives. There is an abundance of research on the technical aspects of UAV systems: their design, implementation, operation, diagnostics, and stability (Moir \& Seabridge, 2012). However, a more holistic approach to ensuring safe operations in a heterogeneous airspace is re-

Marcos Quiñones-Grueiro et al. This is an open-access article distributed under the terms of the Creative Commons Attribution 3.0 United States License, which permits unrestricted use, distribution, and reproduction in any medium, provided the original author and source are credited.

${ }^{1} \mathrm{UAV}$ sales are expected to top $\$ 12$ billion in 2021 (https://www . businessinsider.com/drone-technology-uses-applications quired to address this multi-faceted problem that comprises of component and system diagnostics, system-level prognostics, airspace safety assurance, flight path risk assessment, as well as trajectory planning and replanning, just to name a few (Clothier \& Walker, 2006).

Our overall goals is to support safe operations of package delivery UAVs operating in urban environments. We have developed a decision-making framework to maintain system safety during a UAV mission (flight from a starting point to a destination going through a sequence of pre-determined way points) by minimizing the overall risk of mission failure, considering a number of risk factors along with uncertainties in the environment and the operating state of the vehicle (UAV). In this paper, we develop a Risk analysis approach that computes and updates risks associated with projected UAV flight paths by considering two potential hazards:(1) collision (with static obstacles), and (2) depleting battery charge below a pre-specified safe threshold. In addition, we also take into account the effects of degradation in system components on overall UAV flight. In general, multiple components of a system may degrade at the same time, therefore, we develop methodologies for computing system performance using a system-level prognostics approach that we have developed in past work (Khorasgani et al., 2016).

A primary task for ensuring safe UAV flight operations requires careful flight planning and trajectory generation based on a series of four-dimensional waypoints (latitude, longitude, altitude, and arrival time), while satisfying a set of constraints that assure safety during flight (e.g., distance from nearest obstacle $\geq 2 m$, battery charge $\geq 10 \%$, risk threshold $\leq 15 \%$ ), and optimizing a set of performance parameters (e.g., flight time, power consumption). We assume our package-carrying UAV is a low flying craft that typically flies over roads between large buildings. Our path planning algorithm assumes the existence of a map of possible routes that 
UAVs can fly within this urban environment. Trajectory generation involves the process of deriving a stable trajectory, defined by a set of waypoints, with the assumption that the $\mathrm{UAV}$, if undisturbed will fly a straight line path between two consecutive way points. The UAV trajectory is smoothed using B-splines at corners, implying that the UAV does not have to make sharp turns at corners.

In situations where there are no ambient disturbances, such as wind direction changes or wind gusts, we assume the UAV chooses the minimum distance path between its start and destination points, because that consumes the least amount of battery charge (i.e., energy), thus minimizing risk of crash due to loss of power. However, wind gusts and changes in environmental conditions in the vicinity of where the UAV is flying, may result in a change in flight risk, causing it to exceed specified thresholds. In extreme circumstances, the UAV may decide that it is too risky to continue flying, and, invokes emergency landing procedures instead. We do not deal with contingency management in this paper.

The rest of this paper is organized as follows. Section 2 reviews the literature on UAV safety analysis. Section 3 provides a description of of our octocopter UAV system model and the test bed for our experimental studies. Section 4 presents our decision making framework. Section 5 presents the system-level prognostics scheme considered in this paper. The results of our experimental case studies run on an octocopter system under different scenarios are presented in Section 6. Finally, the conclusions of our work and directions for future research are presented in Section 8.

\section{BaCkground: Safety Analysis, Health Man- agement and Path Planning for UAvs}

Past work on UAV safety analysis has focused on collisions of these systems with other aircraft flying in the civil airspace with recommendations on developing and advancing Traffic Collision and Alert Systems (TCAS) that can be deployed to minimize these collisions (Kuchar, 2005). Clothier \& Walker (2006) contend that overall safety requirements for UAV systems should be the same as that for human-piloted aviation. They developed a simple simple fatality model to illustrate the impact of different safety objectives on the design and operation of UAV systems. They used comparative examples to highlight the importance of the nature of risk exposure to the type of operation being performed.

A number of studies have also characterized UAV flight safety or risks associated with the potential harm to humans and property that would result from UAV crashes to the ground. In this regard, Atkins (2014) introduced the concept of geofencing in the context of safe UAV flights for agricultural applications, such as crop inspections and insecticide spraying. Ippolito (2019) generalized and extended the notion of safety analysis to dynamic ground risk mitigating flight control by analyzing methods for (1) reducing the likelihood of occurrence (e.g., flight planning to avoid overpopulated areas) and (2) reducing the severity of the consequences when operating in low altitude, high density urban areas. The author discussed a number of risk mitigation strategies that include (1) physical separation, (2) geofencing around important assets, (3) fault monitoring, and (4) safe flight termination in case of contingencies to address risk mitigation control of UAV systems.

Recently, a number of authors have developed fault detection and prognostics approaches to support risk mitigation and increase safety of UAV flights. For example, Saha (2007) and Sierra, et al (2019) discuss approaches for tracking UAV battery health during operations. Balaban \& Alonso (2013) propose modeling techniques based on Partially Observable Markov Decision Processes (POMDPs) for prognostic decision making. POMDPs allow for representation of uncertainties in state estimation (including in payoffs/rewards), action outcomes, and future operating conditions. They apply this approach to a mission replanning case study for UAVs.

Traditionally, approaches developed in robotics have evaluated risk without considering the state of the vehicle. For instance, data driven approaches to risk analysis have taken into account uncertain weather conditions (Rubio-Hervas et al., 2018) and measurement uncertainty (De Filippis et al., 2011) together with the vehicle's kinematics, but they have ignored the fact that a UAV's operating conditions can change during flight. On the other hand, contributions from the field of control systems incorporate trajectory planners that consider the dynamic model of the vehicle (Brown \& Rogers, 2016), but do not take into account the varying environmental conditions for risk analysis. However, in urban scenarios, it is important to consider the interactions between the system's state and the varying environmental conditions (Coutinho et al., 2018). Computing risk and safety is another area of research that is a key component of ensuring safe UAV operation. Lin \& Shao (2020) compute the expected level of safety (ELS) of a path as a function of mean time between failures, the area of exposure in square meters (assuming a ground impact), the population density, and accident severity.

Several recent studies have demonstrated promising methods for fast trajectory generation. In Corbetta et al. (2019), the authors use B-spline curves to generate a smooth trajectory given a set of 4D waypoints. In Schopferer \& Benders (2020), the authors use a multi-objective cost function that optimizes flight time, altitude, and risk. They quantify risk based on collision with objects (such as buildings), and pre-compute a search graph. During replanning, K-Nearest-Neighbors is utilized to reduce the search space and the $\mathrm{A}^{*}$ algorithm is used to find a new path within that search space. In Primatesta et al. (2019), the authors compute a discretized risk-map that associates a given cell on the map with a given level of 
risk based on population density, and utilize Dubins curves to generate a smooth trajectory.

In this paper, we take into account the uncertainty in weather conditions (e.g., wind speeds, wind gusts) and the health state of a UAV in analyzing risk and safety of low-altitude UAV flights in urban areas. We discuss our UAV system and the environment in which it operates next, and then discuss our approach to risk analysis for safe flights.

\section{SYSTEM DESCRIPTION}

In this section, we describe the octocopter airframe model, its subsystems, and the control system responsible for flying the UAV.

\subsection{UAV Model}

The octocopter airframe dynamics are modeled based on Newton-Euler equations of motion for a rigid body (Valavanis \& Vachtsevanos, 2015). The octocopter if composed of a central hub with 8 arms extending radially and a motor at the end. The derived body forces considered are

$$
F_{b}=F_{M}+F_{D}+m g R_{I B} \mathbf{e}_{z},
$$

where $F_{b} \in \Re^{3}$ is the resulting force acting on the body frame, $F_{M} \in \Re^{3}$ is the resultant force generated by the motors, $F_{D} \in \Re^{3}$ is the drag force resulting due to the movement of a UAV through the air, $m$ is the mass of the UAV, $g$ is the gravity acceleration, $R_{I B} \in \Re^{3 \times 3}$ is the rotation matrix from the inertial frame to the body frame, and $\mathbf{e}_{z}=\left[\begin{array}{lll}0 & 0 & 1\end{array}\right]^{T}$.

The rotation matrix is calculated based on the Euler angles $[\phi, \theta, \psi]$ (Mahony et al., 2012). The order of rotations from the inertial frame to the body frame considered in this work is yaw (Z)-pitch (Y)-roll (X) assuming the UAV is moving forward in the positive $\mathrm{X}$ direction. The equations of motion derived from equation (1) are

$$
\begin{array}{r}
\dot{\mathbf{v}}_{b}=\frac{1}{m}\left(F_{M}+F_{D}\right)+g R_{I B} \mathbf{e}_{z}-\mathbf{w}_{b} \times \mathbf{v}_{b} \\
\dot{\mathbf{w}}_{b}=I_{b}^{-1}\left(M_{M}-\mathbf{w}_{b} \times I_{b} \mathbf{w}_{b}\right),
\end{array}
$$

where $\mathbf{v}_{b} \in \Re^{3}$ and $\mathbf{w}_{b} \in \Re^{3}$ represent the linear and angular velocity vectors of the UAV in the body frame, $M_{M}$ is the torque generated by the motors, and $I_{b} \in \Re^{3 \times 3}$ is the inertial matrix of the UAV. Next, we describe how each force and torque is calculated.

\subsubsection{Motor forces and torques}

The eight rotating motors including propellers are used to generate motor forces $F_{M}$ and torques $M_{M}$. The disposition and rotation of the motors is shown in Figure 1. For each motor $i$, the force and torque generated are given by

$$
\begin{gathered}
F_{M_{i}}=c_{T} \omega_{M_{i}}^{2} \\
M_{M_{i}}=c_{Q} \omega_{M_{i}}^{2},
\end{gathered}
$$

where $c_{T}$ is the coefficient of thrust and $c_{Q}$ is the torque constant. Both parameters can be easily estimated from static thrust tests (Mahony et al., 2012). The net force applied to the airframe is the summation of the forces generated by the motors. The net torque acting on the octocopter arises from the aerodynamics (the combination of the produced rotor forces and air resistances) applied to the $\mathrm{N}$-rotor vehicle.

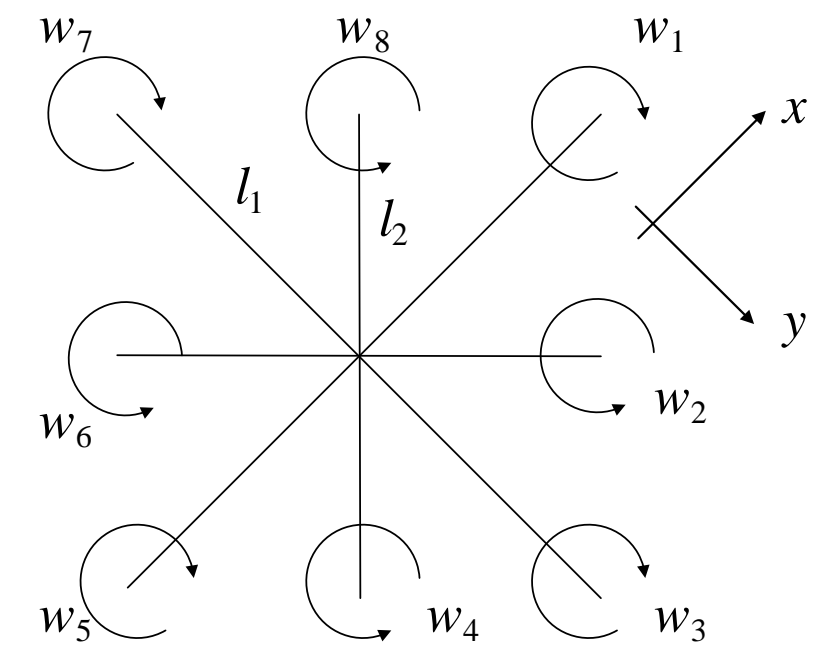

Figure 1. The octocopter seen from above. Showing the numbering of each motor and their rotational directions

\subsubsection{Drag force}

Form drag is the most common and easily modeled aerodynamic effect. Form drag arises due to the movement of a reference area through a fluid. The general expression of the form drag force is

$$
F_{\text {Drag }}=-\frac{1}{2} \rho C_{D} A \mathbf{v}_{b}^{2},
$$

where $\rho$ is the air density, $C_{D}$ is the drag coefficient, $A$ is the reference area that is perpendicular to the velocity of the object $\left(\mathbf{v}_{b}\right)$. Drag force generated due to translation of the octocopter is given by

$$
F_{D}=-\frac{1}{2} \rho C_{D}\left(\begin{array}{c}
A_{y z} v_{b, x}\left|v_{b, x}\right| \\
A_{x z} v_{b, y}\left|v_{b, y}\right| \\
A_{x y} v_{b, z}\left|v_{b, z}\right|
\end{array}\right)
$$

where $|$.$| is the absolute value (necessary since the aerody-$ namic drag always act in opposite direction of the velocity vector), $A$ represents the cross-sectional area of the UAV in 
each plane, and $v_{b, i}$ represents the velocity of the $i$ axis in the body frame. The torque generated due to drag is considered negligible in this work.

\subsubsection{Wind effect}

Wind is a major disturbance source for light aerial vehicles, such as the octocopter. Being able to remain stable and minimize wind disturbances is a requirement for the use of UAVs in urban scenarios. Reactive approaches to wind rejection have been the subject of prior research (Gougeon et al., 2018). Modeling the effect of the wind disturbance is achieved by generating a wind velocity vector varying randomly around a nominal value and transforming this vector into the resulting wind forces and moments acting on the octocopter.

The wind velocity vector is defined by

$$
\mathbf{v}_{w}=\mathbf{v}_{w, n o m}+\mathbf{v}_{w, \text { rand }}
$$

where $\mathbf{v}_{w, n o m}$ is the nominal component and $\mathbf{v}_{w, \text { rand }}$ is the stochastic component. A valid representation of the latter term can be generated by using Dryden's turbulence model (Gougeon et al., 2018). The wind affects the translation of the octocopter by modifying the drag force as follows

$$
\begin{gathered}
F_{\text {Drag }}^{\text {wind }}=\frac{1}{2} \rho C_{D} A \mathbf{v}_{a}^{2} \\
\mathbf{v}_{a}=R_{I B} * \mathbf{v}_{w}-\mathbf{v}_{b},
\end{gathered}
$$

where $\mathbf{v}_{a}$ represents the apparent velocity of the UAV subject to the wind velocity in the inertial frame $\mathbf{v}_{w}$. The change in the moment dynamics caused by the wind acting on the propellers of the motors is assumed to be negligible in this work.

\subsection{Propulsion system}

The propulsion system of the octocopter is formed by a set of BrushLess DC Motors (BLDCM), an Electronic Speed Controller (ESC), and a Lithium Polymer battery. Each motor is powered by the battery through the ESC, which controls the angular speed through a PWM signal. The dynamics of the ESC are neglected in this work. We assume, therefore, that the voltage supply of the battery and the current consumed by the motors are averaged with respect to the duty cycle value of the control signal.

\subsubsection{Motor model}

A BrushLess DC motor is a type of permanent magnet synchronous motor driven by a DC supply voltage. The main feature of this type of motor that makes it suitable for aerial robotic applications is its long operating life and high torqueweight ratio. The mathematical model that describes the dynamics of the angular speed of each motor can be described by the following equations

$$
\begin{array}{r}
\dot{\omega}_{i}=\frac{1}{J_{m}}\left(K_{e} i_{c}-T_{\text {load }}-D_{f} \omega-T_{f}\right), \\
i_{c}=\frac{1}{R_{e} q}\left(v_{D C}-K_{e} \omega_{i}\right),
\end{array}
$$

where $R_{e} q=\frac{2}{3} \sum_{j=1}^{3} R_{j}$ is the equivalent electric resistance of the coils, $K_{e}$ is the back electromotive force constant, $\omega_{i}$ is the angular velocity of the $i$ th BLDCM, $T_{f}$ is the static friction torque, $D_{f}$ is the viscous damping coefficient that allows to estimate the dynamic friction torque $\left(D_{f} \omega\right), J_{m}$ is the inertia of the BLDCM, $v_{D C}$ is the input voltage control signal, $i_{c}$ is the current demanded from the battery pack, and $T_{\text {load }}$ represents the torque load generated by the propellers. The first equation represents the mechanical dynamics and the second one the electrical dynamics. The relationship between the torque load and the angular velocity is non-linear and can be defined from experimental data according to the dimensions and material of the propellers.

\subsubsection{Battery model}

Lithium Polymer (LiPo) batteries transform the energy released by spontaneous chemical reaction to electricity work. LiPo batteries have desirable properties, such as high discharge rate (C-rate), high energy, and power densities. This has motivated their use for electric aerial vehicles. A LiPo battery pack is formed by individual cells connected to each other in series or in parallel (Julien et al., 2016).

The electric dynamics of a battery cell can be modeled through an Equivalent Circuit Representation. The output voltage of the battery $V_{b a t}$ will depend on the open circuit voltage (effective voltage that depends on the state of charge $(S o C)$ of the battery), the diffusion voltage (transitory response of the voltage given an input current), and the voltage that characterizes the ohmic over-potential due to the internal resistance of the battery. The state of charge of the battery represents the proportion of the charge available at a given time compared to the total charge available when the battery is fully charged $(S o C \in[0,1])$. The equations that characterize the behavior of the battery considering the effects mentioned above are the following

$$
\begin{gathered}
\dot{V}_{S o C}=-\frac{i_{c}}{Q} \\
\dot{i}_{d}=\frac{i_{c}}{R_{d} C_{d}}-\frac{i_{d}}{R_{d} C_{d}} \\
V_{\text {out }}=V_{\text {ocv }}-R_{d} i_{d}-R_{0} i_{c}
\end{gathered}
$$

where $Q$ represents the total capacity of the battery, $i_{c}$ the input current, $R_{d}$ and $C_{d}$ are the diffusion resistance and capacitance, $i_{d}$ the current going through the diffusion resis- 
tance, $R_{0}$ represents the internal resistance, $V_{\text {ocv }}$ is the open circuit voltage, and $V_{\text {out }}$ is the output voltage of the battery. The open circuit voltage depends non-linearly on the state of charge of the battery. The function that characterizes such relationship can be experimentally estimated and approximated as a polynomial by using charge and discharge data.

\subsection{Control scheme}

The hierarchical control scheme implemented for the octocopter is shown in Figure 2. This control approach is commonly used for multicopters since it allows for stabilization of the position and orientation of the octocopter with respect to a trajectory. The low level is in control of vehicle attitude, and the top level is in control of position along a trajectory forming nested feedback loops.

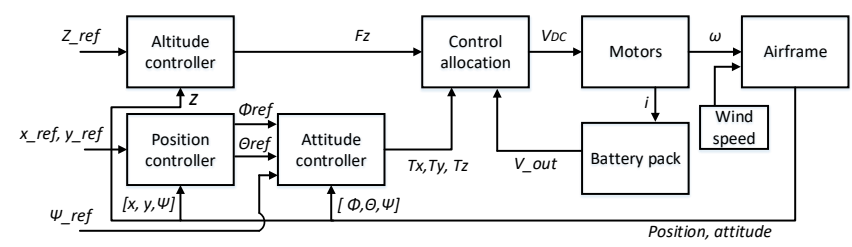

Figure 2. Hierarchical control scheme for the octocopter

The reference trajectory for the octocopter can be defined for each time step in terms of position and yaw angle $\left[x_{r e f}, y_{r e f}, z_{r e f}, \psi_{r e f}\right]$. Altitude is controlled through a Proportional Integral Derivative (PID) controller based on the reference input. This controller generates the required force in the $z$ direction $\left(F_{z}\right)$. The position controller block estimates, based on the current position of the vehicle and yaw angle, the reference for the pitch $\left(\theta_{\text {ref }}\right)$ and roll $\left(\phi_{\text {ref }}\right)$ angles. The attitude controller block is formed by three PID controllers for pitch, roll, and yaw $(\theta, \phi, \psi)$. This block generates the required torque in each direction $\left(T_{x}, T_{y}, T_{z}\right)$. The control allocation block transforms the required torques and force into a reference voltage $\left(V_{D C}\right)$ for each BLDC motor of the octocopter based on the maximum voltage that the battery can provide $\left(V_{\text {out }}\right)$. Finally, the motor block generates the angular velocities according to the motor's dynamics $(\omega)$. These angular velocities together with the wind vector are used to calculate the forces and torques acting on the body frame according to the equations of motion described before.

\subsection{Degradation of Components}

The state of health of a component describes the actual physical condition in comparison with its nominal condition. The degradation of the components of the octocopter from mission to mission affects their respective state of health as well as the state of health of the octocopter. The motors, battery, and sensors of the octocopter are susceptible to degradation mechanisms that eventually may cause the failure of the system.

BLDC motors are susceptible to mechanical degradation in the form of general motor or bearing wear, and less common electrical degradation in the form of contact corrosion and insulation deterioration (Abramov et al., 2014). An increase in winding resistance results in increased power consumption under load (and decreased consumption under no-load conditions), as depicted in the FMECA chart provided in (Kulkarni $\&$ Corbetta, 2019). The mechanical degradation is associated with the static and dynamic friction parameters $\left(T_{f}\right.$ and $\left.D_{f}\right)$ of the motor model presented before. The winding resistance change is associated with the electric resistance of the coils $\left(R_{e} q\right)$.

The state of health of the battery decreases with time due to aging mechanisms caused by the charge/discharge cycles and the damage due to deep discharges. The aging of the battery causes an energy loss leading to capacity loss (a reduction of battery capacity), and the increase of the battery impedance (power fade). Capacity loss is evidenced in the battery model by the reduction of the total capacity parameter $Q$, and the power fade by the increase of the internal resistance $R_{0}$ [33].

\section{Decision-Making Framework For SAFETy ANALYSIS}

Our goal to maintaining system safety during a mission will be to minimize overall risk of failure by considering a number of risk factors along with uncertainties in the environment and operating state of the vehicle (UAV). A mission plan is defined in terms of a set number of way-points $C=$ $\left\{c_{1}, c_{2}, \ldots, c_{n}\right\}$ and the desired cruise speed. We will consider mission failure to be caused by two factors: (1) the loss of control of the vehicle resulting in a collision with other objects or (2) a crash into the ground. Another reason for mission failure is when the remaining useful life (RUL) of the UAV is smaller than the time required to complete the mission. The primary causes of mission failure considered in this work are: (1) adverse weather conditions and/or (2) system performance degradation. System performance degradation might be caused by the degradation or fault of different components of the UAV, such as the battery, the motors, and the Inertial Measurement Unit.

Let $G$ be a graph where the set $V$ represents all the way-points that must be visited by the UAV and $A$ represents the set of arcs between way-points. The optimization problem can be formalized as follows:

$$
\min _{\Gamma_{i j} \in \Gamma_{i j}} f_{m f a i l}\left(\Gamma_{i j}\right)
$$

where $\Gamma_{i j}$ represents a trajectory between way-point $c_{i}$ and $c_{j}, \Gamma_{i j}$ represents the set of possible trajectories between the two way-points, and the function $f_{m f a i l}$ estimates the proba- 
bility of mission failure. In the context of dynamic systems, the optimization problem above is subject to the following constraints:

- Dynamics of the system:

$$
\mathbf{x}_{n+1}^{i j}=f\left(\mathbf{x}_{n}^{i j}, \mathbf{u}_{n}^{i j}, \vartheta_{n}^{i j}, \mathbf{w}_{x}^{i j}\right),
$$

where $\mathbf{x}_{n}, \mathbf{u}_{n}, \vartheta_{n}$, and $\mathbf{w}_{x}$ represent the set of variables that characterize the dynamics of the UAV, the inputs of the system, the degrading parameters of the system components, and the uncertainties associated with the system model at time step $n$, respectively. This constraint means that the system dynamics must be satisfied between any two way-points $i$ and $j$ to be flown.

- Bounded risk of collision:

$$
F_{c}^{\max }\left(\Gamma_{i j}\right)<\tau_{c},
$$

where $F_{c}$ represents the maximum probability of mission failure because of collision with an obstacle along the trajectory $\Gamma_{i j}$.

- Bounded remaining useful life:

$$
F_{\text {rul }}\left(\Gamma_{i j}\right)<\tau_{\text {rul }},
$$

where $F_{r u l}$ represents the probability of mission failure because of the RUL being smaller than the time required to complete the mission.

The risk of mission failure function is defined as follows

$$
f_{\text {mfail }}\left(\Gamma_{i j}\right)=\max \left(F_{c}\left(\Gamma_{i j}\right), F_{\text {rul }}\left(\Gamma_{i j}\right)\right)
$$

Instead of trying to solve a multi-objective optimization problem which might not be tractable, here we minimize the maximum of the two risks considered. In the next subsections, we describe how each of these terms is computed.

\subsection{Risk of collision}

Risk analysis conducted on projected UAV flight paths enables the safe operations of these vehicles in urban scenarios. Total risk of collision of a mission is accumulated along the respective trajectory by considering a set of $K$ static obstacles in the environment. The average risk can be calculated according to

$$
\operatorname{Risk}_{t o t}=\frac{1}{K} \sum_{k=1}^{K} \int_{\Gamma} f\left(\Gamma, \text { obst }_{k}\right) d \Gamma,
$$

where $f\left(\Gamma\right.$, obst $\left._{k}\right)$ is a function that calculates the probability of collision of the UAV with obstacle $k$ when it follows trajectory $\Gamma$. In order to solve this equation, the trajectory of the UAV must be expressed in a discrete form by considering a sampling time thus the total risk can be expressed as follows

$$
\operatorname{Risk}_{n}^{\text {tot }}=\frac{1}{K N} \sum_{k=1}^{K} \sum_{n=1}^{N} f\left(\Gamma(n), \text { obst }_{k}\right),
$$

where $\Gamma(n)$ represents the position of the UAV at time $n$, and $f\left(\Gamma(n)\right.$, obst $\left._{k}\right)$ takes the following form

$$
f\left(\Gamma(n), \text { obst }_{k}\right)=p\left(D_{\text {obst }_{k}}^{n}<d s a f e_{k}\right),
$$

where $D_{o b s t_{k}}^{n}$ represents the distance between the UAV at time $n$ of the trajectory and the obstacle $k$, and $d s a f e_{k}$ represents a minimum distance separation that must be kept between the UAV and the obstacle. We assume the position of the UAV at time $n$ as a stochastic variable such that the variable $D_{\text {obst }_{k}}$ is also stochastic and follows a normal distribution, $D_{\text {obst }_{k}} \sim \mathcal{N}(\mu, \sigma)$. This equation evaluates the inverse cumulative distribution function of $D_{o b s t_{k}}$ at $d s a f e_{k}$. The parameters of this distribution are estimated by propagating the estimated uncertainties of the UAV position variable through the reference trajectory.

The position of the UAV is represented by the coordinates of its center of mass. Assuming a two dimensional map, we consider a set of static obstacles that can be represented as a rectangle defined in terms of four coordinates in a plane obst $_{i}=(x, y), i=1, \ldots, 4$. For a realization of the trajectory, the distance is computed as $d_{o b s t_{k}}=\left|\xi-\nu_{k}\right|$, where $\xi$ is a vector of the coordinates of the UAV and $\nu_{k}$ is the closest vertex of obstacle $k$. The distance threshold dsafe $e_{k}$ must be empirically defined to account for the dimensions and orientation of the UAV, and the errors introduced in the discretization of the obstacle.

Another practical measure to consider is the maximum risk of collision along a trajectory defined by

$$
\operatorname{Risk}_{n}^{\max }=\max _{n \in N} \sum_{k=1}^{K} f\left(\Gamma(n), \text { obst }_{k}\right)
$$

We calculate $F_{c}$ as $R i s k_{t o t}$ and $F_{c}^{\max }$ as $R i s k_{\max }$.

\subsection{Safety Analysis}

Safety analysis is based on system-level prognostics and it encompasses two distinct but related problems:(1) estimating the current system state and the degradation rates of individual components; and (2) predicting future system performance by deriving system RUL functions.

For our system-level prognostics approach, we follow an analytic framework that tracks the degradation rate of individual components, such as the battery and motors from mission to mission. Based on the degradation parameters estimated, we use stochastic simulations to compute and predict the change in system performance, e.g., the ability to maintain a specified trajectory over time. The system-level prediction problem is 
designed to compute the system $R U L_{n}$ distribution function at time instant $n$ defined by

$$
R U L_{n}=\left(E O L_{n}-n\right) \Delta t,
$$

where $\Delta t$ is the sampling time of the system, $n \in N$ represents the sampling step, and $E O L_{n}$ represents the end of life of the system defined by

$$
E O L_{n}=\inf \left\{z \in Z: z \geq n \& T\left(\wp_{z}\right)=1\right\}
$$

where $T\left(\wp_{z}\right)$ is the following system performance threshold function

$$
T\left(\wp_{z}\right)= \begin{cases}1 & \text { if } 0 \in R\left(\wp_{z}\right) \\ 0 & \text { if } 0 \notin R\left(\wp_{z}\right)\end{cases}
$$

$R\left(\wp_{z}\right)$ denotes a set of performance constraint functions that map the performance of the system to a Boolean domain $[0,1]$. A value of 1 implies that the constraint is satisfied, and a value of 0 implies that it is not. Such set of constraints is defined based on the system degradation model.

Definition 4.1 (System degradation model) System degradation model of a system is given by

$$
\begin{gathered}
\mathbf{x}_{n+1}=f\left(\mathbf{x}_{n}, \mathbf{u}_{n}, \vartheta_{n}, \mathbf{w}_{x}\right) \\
\varrho_{n+1}=g\left(\varrho_{n}, \alpha_{n}, \mathbf{x}_{n}, \mathbf{w}_{d}\right) \\
\mathbf{y}_{n+1}=h\left(\mathbf{x}_{n}, \mathbf{u}_{n}, \vartheta_{n}, \mathbf{w}_{y}\right),
\end{gathered}
$$

where $n$ represents the current time step, $\vartheta_{n}$ is the set of degrading parameters, $g$ is the set of degradation functions, $\alpha_{n}$ is the set of degradation model parameters in the system, $\mathbf{w}_{x}$, $\mathbf{w}_{d}$, and $\mathbf{w}_{y}$ capture the system model uncertainties, the set of uncertainties in the degradation models, and the measurement noise, respectively.

The risk of not completing the mission can be calculated at each time step $n$ as follows

$$
F_{\text {rul }}\left(\Gamma_{i j}\right)=p\left(R U L_{n}<t_{\text {end }}\right),
$$

where $t_{\text {end }}=N * \Delta t$ represents the time planned for the UAV to reach the final way-point.

\section{SYSTEM-LEVEL PROGNOSTICS METHODOLOGY}

We follow the system-level prognostics methodology proposed in Khorasgani et al. (2016). Figure 3 presents the methodology, which combines the estimation of system states, parameters, and degradation parameters, with a prediction strategy to compute the risk of mission failure functions at each time step $n$.

Different state estimation methods can be used to estimate the probability density function of the states and parameters of the UAV and its components. The estimation of the attitude, velocity, and position has been accomplished through complementary filtering techniques (Mahony et al., 2012), Kalman filtering methods and its extensions (Burri et al.,

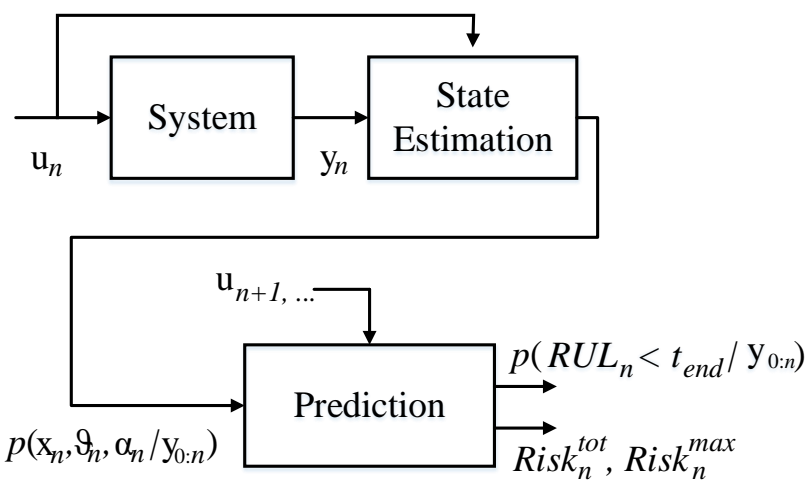

Figure 3. System level prognostics methodology

2015; Yang et al., 2017; Al-mashhadani, 2019). Inertial and visual sensor fusion algorithms can be employed in case visual sensors are available (Corke et al., 2007). The state estimation of the motors is performed in this work through the extended Kalman filter since non-linearity is smooth. Numerous methods have been proposed for the estimation of the states and parameters of the battery, i.e. unscented Kalman filter, particle filter, etc (Plett, 2015; Sierra et al., 2019). We considered implementations of the unscented Kalman filter for tracking the attitude and position of the UAV (Merwe et al., 2004), as well as for tracking the state variables of the battery (Kulkarni et al., 2018).

The distribution of $R U L_{n}$, and the accumulated and maximum risk $\left(\right.$ Risk $_{n}^{\text {tot }}$, Risk $\left._{n}^{\max }\right)$ associated with the current trajectory are calculated though stochastic simulations considering the distribution of the system states and parameters $p\left(\mathbf{x}_{n}, \vartheta_{n}, \alpha_{n} \mid \mathbf{y}_{n}\right)$, the distribution of future inputs to the system, the distribution of the system model uncertainties and the uncertainties in the degradation models at each time step $n$. We therefore generate multiple samples in a Monte Carlo fashion representing the system, and and simulate each one to the end of the trajectory and to the system's end of life.

\section{EXPERIMENTAL STUdY}

We will demonstrate the feasibility of the proposed decisionmaking framework for evaluating three alternative trajectories between two way-points. Figure 4 shows a map of the obstacles in the environment as well as the staring point, the goal, and the three trajectories considered for evaluation. We test the proposed decision making framework in an offline setting, which includes the prediction step of the system-level prognostics methodology.

Uncertainty propagation is performed in a Monte Carlo fashion by simulating the behavior of the octocopter system described in Section 3 along the initial reference trajectory. For the $j$ th Monte Carlo trajectory realization, we sample from 


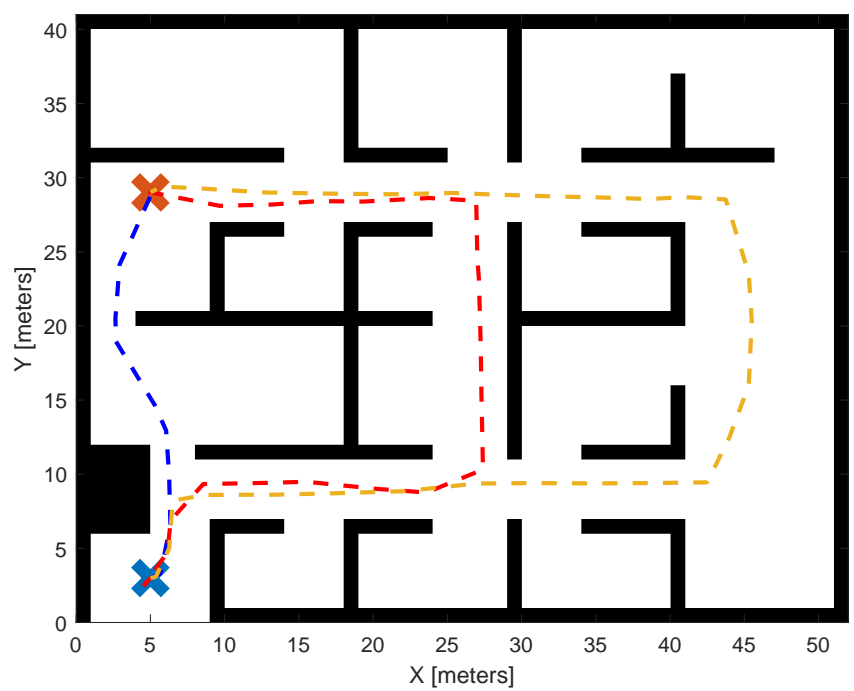

Figure 4. Map of obstacles with three possible trajectories between two way-points

the initial distribution of states to set up the initial condition and record the states of the UAV with respect to the reference path. Thus, a set of $M$ trajectories are collected, where $M$ is the number of Monte Carlo samples. The two sources of uncertainty considered for this experiment are noise affecting the sensors, and the random component of the wind vector presented in equation 8 .

We consider three test scenarios: (1) nominal flight conditions, (2) aggressive wind conditions, and (3) the degradation of one of the BLDC motors. The performance test defining the EOL of the UAV is determined by the state of charge of the battery becoming smaller than $20 \%$. Other system-level performance tests can be defined but here we just use this to demonstrate how the proposed decision making framework can be used. Figure 5 presents three realizations of the trajectory under nominal conditions. From a safety analysis perspective, the three trajectories present a low risk of collision and a low risk of the RUL being smaller than the time required to complete the mission. In this case, other criteria can be used to select the best trajectory. Figure 6 shows the RUL at the end of each trajectory. The blue trajectory is completed in less time thus the RUL at the end is larger than for the other trajectories. This means that by following the blue trajectory the octocopter can complete more missions before reaching the EOL condition.

Figure 7 presents three realizations of the trajectory under aggressive wind conditions (nominal speed of $11 \mathrm{~m} / \mathrm{s}$ and wind gusts with maximum speed of $0.5 \mathrm{~m} / \mathrm{s}$ ). The wind is simulated in the positive $x$ axis direction. Figure 8 shows the risk of collision with the closest obstacle at each time step of each trajectory. We considered $s a f e_{k}=1 \mathrm{~m}$. The average risk $\left(\right.$ Risk $\left.^{t o t}\right)$ is $0.096,0.1228$, and 0.0446 for the blue,

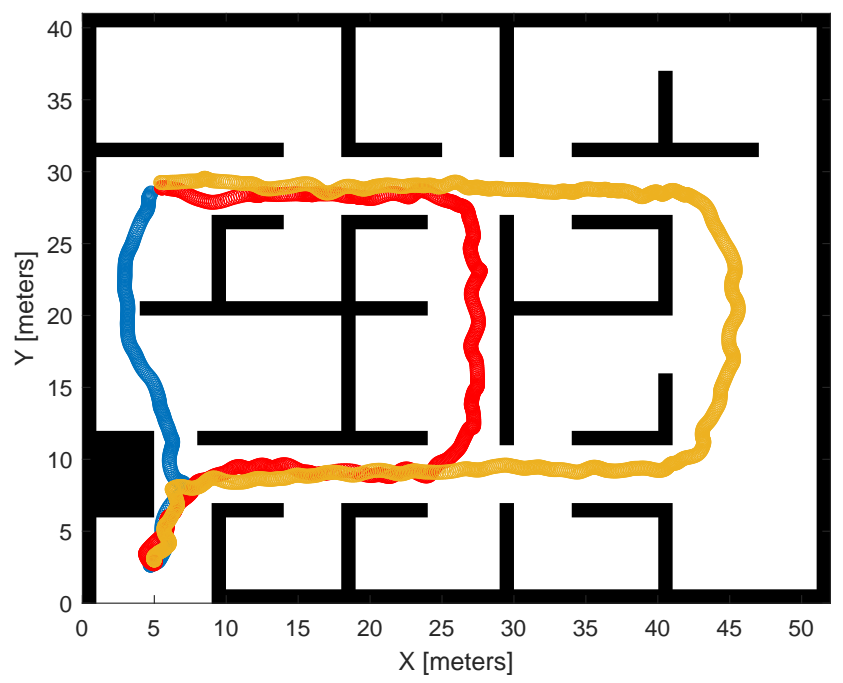

Figure 5. Example of the three trajectories under nominal conditions

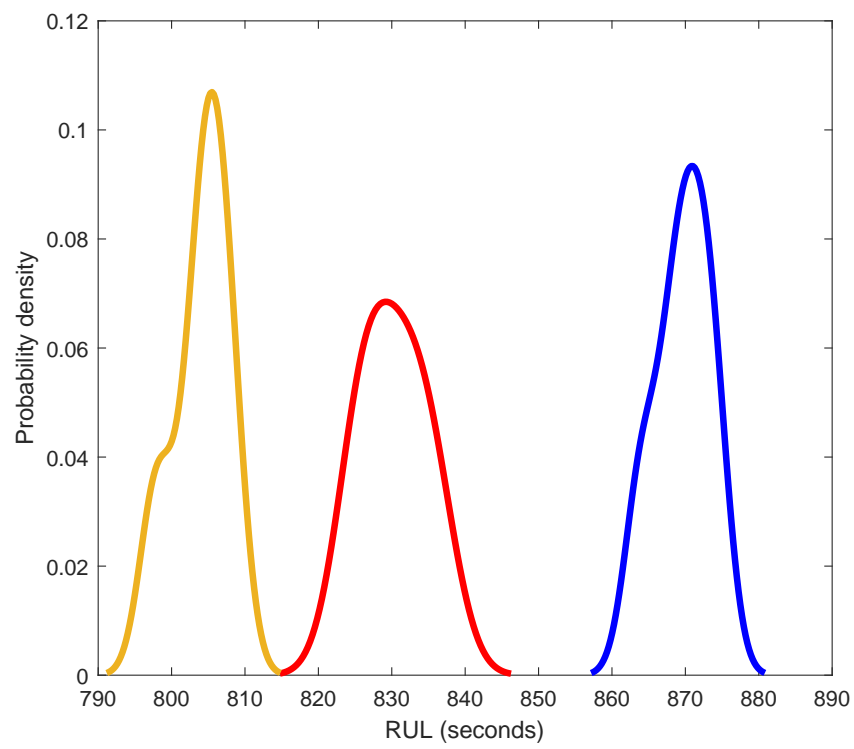

Figure 6. Remaining useful life for the octocopter at the end of each trajectory 


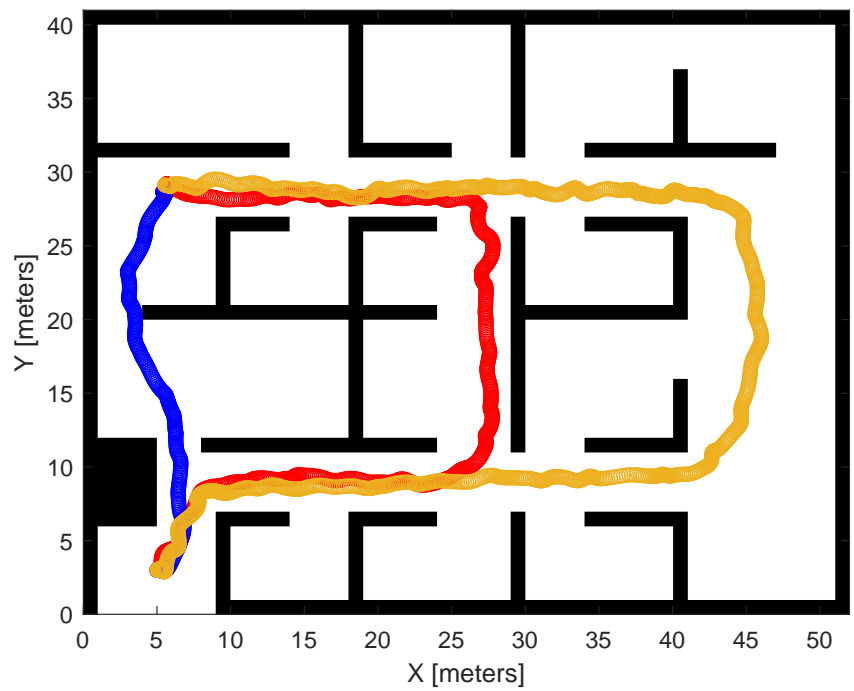

Figure 7. Example of the three trajectories under aggressive wind conditions

red, and yellow trajectory, respectively. The maximum risk $\left(\right.$ Risk $\left.^{\max }\right)$ is $0.7539,0.7096$, and 0.3037 for the blue, red, and yellow trajectory, respectively. These results show that while the blue trajectory is more unsafe than the red one on average, the maximum risk of collision is higher for the former one. The yellow trajectory is the safest one in terms of risk of collision.

Figure 9 presents three realizations of the trajectory under the degradation of one of the BLDC motors. The degradation consists of the increase in the coil resistance which results in a loss of power for motor 2 (see Figure 1). This degraded component will affect the flight performance of the octocopter as it can be appreciated in Figure 10. The average risk $\left(\right.$ Risk $\left.^{\text {tot }}\right)$ is $0.2267,0.2035$, and 0.099 for the blue, red, and yellow trajectory, respectively. The maximum risk $\left(\right.$ Risk $\left.^{\text {max }}\right)$ is $0.9697,0.9705$, and 0.8686 for the blue, red, and yellow trajectory, respectively. These results show that the yellow trajectory is the safest on average and also with respect to the maximum risk. However, it is not safe to fly under these conditions given that the maximum risk of collision is high.

\section{Discussion}

Risk assessment for mission planning has been widely studied in recent years. Risk of mission failure is linked to different causes, such as uncertain weather conditions (RubioHervas et al., 2018), measurement uncertainty (De Filippis et al., 2011), and collision with obstacles (Brown \& Rogers, 2016). The novelty of our risk assessment method is that adopts a holistic approach and integrates the different risk factors with the state of health of the UAV. This is especially important in urban scenarios where the interactions between
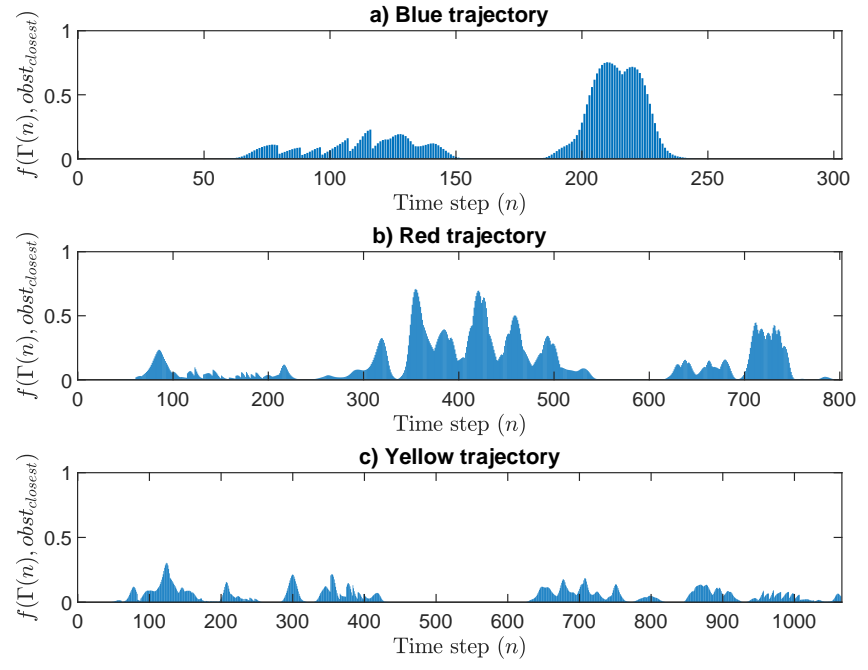

Figure 8. Risk of collision with the closest obstacle at each time step under aggressive wind conditions

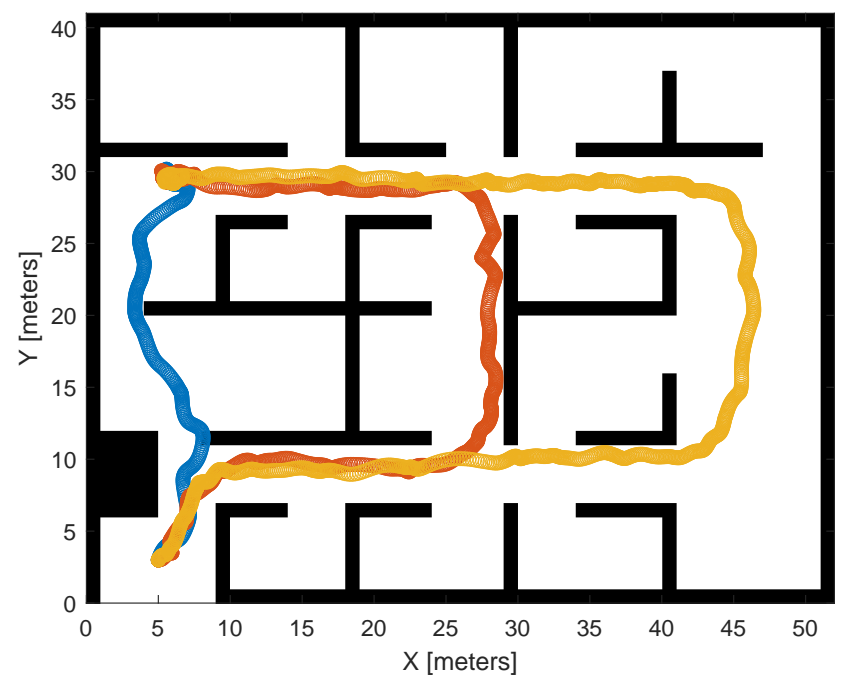

Figure 9. Example of the three trajectories under the degradation of a BLDC motor 

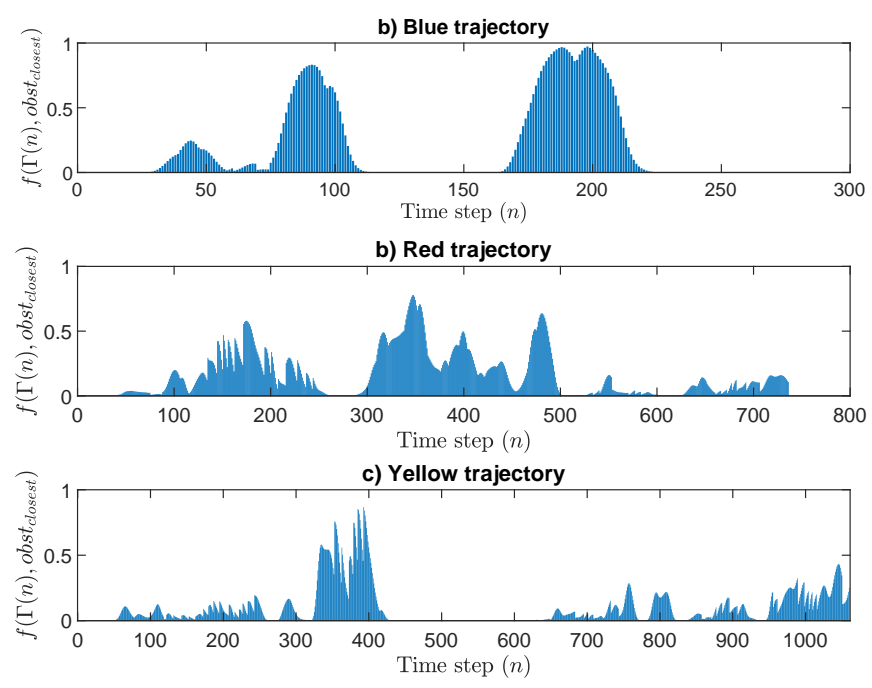

Figure 10. Risk of collision with the closest obstacle at each time step under the degradation of a BLDC motor

the environment and the UAV play an important role in determining the risk of mission failure. The risk to people on the ground is another safety measure that has been widely studied. Capristan and Foster (2017) propose a framework for assessing the threat to people by calculating the potential impact area and the effects of the impact in real-time. Primatesta et al. (2019) propose a path planning strategy that tries to enforce flight operations over low density population areas. The framework we propose can seamlessly integrate with these proposals because the risk to people in the ground can be calculated according to the predicted time of mission failure and the population density in the estimated impact area.

Our decision-making framework is applied to offline trajectory selection, which is determined before UAV commences its mission, therefore, Monte Carlo simulations can be considered to estimate the distribution of the stochastic variables. However, optimal decision making in real time is a more complex problem. For real time assessment of the risk of mission failure, a state estimation-state prediction approach can be used. Online trajectory re-planning requires the use of optimization methods in real time. Previous work has studied the suitability of classic methods like rapidly exploring random trees and the $A^{*}$ graph search algorithm for real time path planning (Zammit \& Van Kampen, 2020). The suitability of evolutionary methods for online path planning has also been proposed recently (Krishnan \& Manimala, 2020). Overall, some of these options could be considered depending on how the path planning problem is formalized.

\section{Conclusions and Future Work}

This paper discusses a decision-making framework to support safe urban operations of individual unmanned aerial vehicles
(UAV) flights. The core of the proposed approach includes a module for risk analysis of mission failure that considers both the risk of collision with obstacles and the risk of not completing the mission because of an unsafe operating state of the vehicle. The risk analysis is computed along a trajectory defined by a set of way points by considering a number of factors, such as the uncertainties in the environment and the current operating state of the vehicle. We tested the proposed approach with an octocopter by comparing the risk of mission failure for three trajectories under different conditions. The risk of mission failure increases under aggressive wind conditions or a degraded motor for some trajectories more than for others. The proposed decision-making framework encompasses multiple measures of risk to select the trajectory with lowest risk. In the future, we plan to develop a a path planning and trajectory optimization algorithm to work in conjunction with the proposed framework to minimize the risk of mission failure both for before take-off planning and online trajectory re-planning.

\section{ACKNOWLEDGMENT}

NASA grant 80NSSC19M0166 from the NASA Shared Services Center.

\section{REFERENCES}

Abramov, I., Nikitin, Y., Abramov, A., Sosnovich, E., \& Bozek, P. (2014). Control and diagnostic model of brushless dc motor. Journal of Electrical Engineering, 65(5).

Al-mashhadani, M. A. (2019). Optimal control and state estimation for unmanned aerial vehicle under random vibration and uncertainty. Measurement and Control, 1-8.

Atkins, E. (2014). Autonomy as an enabler of economicallyviable, beyond-line-of-sight, low-altitude uas applications with acceptable risk. In Auvsi unmanned systems.

Balaban, E., \& Alonso, J. J. (2013). A modeling framework for prognostic decision making and its application to uav mission planning. In Annual conference of the prognostics and health management society (pp. 1-12).

Brown, A., \& Rogers, J. (2016). A sampling-based probabilistic path planner for multirotor air vehicles in cluttered environments. Proceedings of the Institution of Mechanical Engineers, Part G: Journal of Aerospace Engineering, 231 .

Burri, M., Manuel, D., Achtelik, M. W., \& Siegwart, R. (2015). Robust State Estimation for Micro Aerial Vehicles Based on System Dynamics. In Ieee international conference on robotics and automation (icra) (pp. 5278-5283). Washington, D.C.

Capristan, F. M., \& Foster, J. V. (2017). Real-time Risk Assessment Framework for Unmanned Aircraft System (UAS) Traffic Management (UTM). In 17th aiaa avi- 
ation technology, integration, and operations conference (p. 3273).

Clothier, R. A., \& Walker, R. A. (2006). Determination and evaluation of uav safety objectives.

Corbetta, M., Banerjee, P., Okolo, W., Gorospe, G., \& Luchinsky, D. (2019). Real-time uav trajectory prediction for safety monitoring in low-altitude airspace..

Corke, P., Lobo, J., \& Dias, J. (2007). An Introduction to Inertial and Visual Sensing. The International Journal of Robotics Research, 26, 519-535.

Coutinho, W., Battarra, M., \& Fliege, J. (2018, 04). The unmanned aerial vehicle routing and trajectory optimisation problem, a taxonomic review. Computers \& Industrial Engineering, 120.

De Filippis, L., Guglieri, G., \& Quagliotti, F. (2011). A minimum risk approach for path planning of uavs. Journal of Intelligent and Robotic Systems, 61, 203-219.

Gougeon, O., Nguyen, D.-t., \& E, D. S. (2018). Modeling and Control of a Quadcopter Flying in a Wind Field: A Comparison Between LQR and Structured H Control Techniques. 2018 International Conference on Unmanned Aircraft Systems (ICUAS)(1), 1408-1417.

Ippolito, C. A. (2019). Dynamic ground-risk mitigating flight control for autonomous small uas in urban environments. In 2019 aiaa modeling and simulation technologies conference. san diego, ca. jan (Vol. 2109).

Julien, C., Mauger, A., Vijh, A., \& Zaghib, K. (2016). Lithium batteries. In Lithium batteries: Science and technology (pp. 29-68). Cham: Springer International Publishing.

Khorasgani, H., Biswas, G., \& Sankararaman, S. (2016). Methodologies for system-level remaining useful life prediction. Reliability Engineering \& System Safety, 154, 818.

Krishnan, P. S., \& Manimala, K. (2020). Implementation of optimized dynamic trajectory modification algorithm to avoid obstacles for secure navigation of uav. Applied Soft Computing, 90, 106168.

Kuchar, J. K. (2005). Safety analysis methodology for unmanned aerial vehicle (uav) collision avoidance systems. In Usa/europe air traffic management $r \& d$ seminars (Vol. 12).

Kulkarni, C., \& Corbetta, M. (2019). Health management and prognostics for electric aircraft powertrain. AIAA.

Kulkarni, C., Schumann, J., \& Roychoudhury, I. (2018). On-board Battery Monitoring and Prognostics for Electric-
Propulsion Aircraft. In Aiaa/ieee electric aircraft technologies symposium (eats) (pp. 1-12).

Lin, C., \& Shao, P.-C. (2020). Failure analysis for an unmanned aerial vehicle using safe path planning. Journal of Aerospace Information Systems, 1-12.

Mahony, R., Kumar, V., \& Corke, P. (2012). Modeling, Estimation, and Control of Quadrotor. (August).

Merwe, R. V. D., Wan, E. A., \& Julier, S. I. (2004). Sigmapoint Kalman filters for nonlinear estimation and sensorfusion: Applications to integrated navigation. In Aiaa guidance, navigation, and control conference and exhibit (p. 5120). Washington, D.C.

Moir, I., \& Seabridge, A. (2012). Design and development of aircraft systems (Vol. 67). John Wiley \& Sons.

Plett, G. L. (2015). Battery Management Systems Volume 2: Equivalent-Circuit Methods. Artech House.

Primatesta, S., Guglieri, G., \& Rizzo, A. (2019). A riskaware path planning strategy for uavs in urban environments. Journal of Intelligent \& Robotic Systems.

Rubio-Hervas, J., Gupta, A., \& Ong, Y.-S. (2018). Datadriven risk assessment and multicriteria optimization of uav operations. Aerospace Science and Technology, 77, $510-523$.

Saha, B., Goebel, K., Poll, S., \& Christophersen, J. (2007). An integrated approach to battery health monitoring using Bayesian regression and state estimation. In 2007 IEEE Autotestcon (pp. 646-653).

Schopferer, S., \& Benders, S. (2020). Minimum-risk path planning for long-range and low-altitude flights of autonomous unmanned aircraft.

Sierra, G., Orchard, M., Goebel, K., \& Kulkarni, C. (2019). Battery health management for small-size rotary-wing electric unmanned aerial vehicles: An efficient approach for constrained computing platforms. Reliability Engineering \& System Safety, 182, 166-178.

Valavanis, K. P., \& Vachtsevanos, G. J. (2015). In Handbook of unmanned aerial vehicles (chap. Quadcopter Kinematics and Dynamics). Springer.

Yang, H., Lee, Y., \& Dongjun, S.-y. J. (2017). Multi-rotor drone tutorial: systems, mechanics, control and state estimation. Intelligent Service Robotics, 10(2), 79-93.

Zammit, C., \& Van Kampen, E.-J. (2020). Comparison of $\mathrm{a}^{*}$ and rrt in real-time $3 \mathrm{~d}$ path planning of uavs. In Aiaa scitech 2020 forum (p. 0861). 